\title{
Position Control of Ultrasonic Motor Using IMC-PID Combined with Tribes Type NN Algorithm
}

\author{
Shenglin $\mathrm{Mu}^{1, \mathrm{a}}$, Kanya Tanaka ${ }^{1, \mathrm{~b}}$ \\ ${ }^{1}$ Graduate School of Science and Engineering, Yamaguchi University, \\ 2-16-1 Tokiwadai, Ube, Yamaguchi, Japan \\ a p002we@yamaguchi-u.ac.jp, ${ }^{b}$ ktanaka@yamaguchi-u.ac.jp
}

\begin{abstract}
Keywords: ultrasonic motor, IMC-PID control, neural network, particle swarm optimization, parameter-free, tribes-PSO
\end{abstract}

\begin{abstract}
In this paper, we propose a novel scheme of IMC-PID control combined with a tribes type neural network (NN) for the position control of ultrasonic motor (USM). In this method, the NN controller is employed for tuning the parameter in IMC-PID control. The weights of NN are designed to be updated by the tribes-particle swarm optimization (PSO) algorithm. This method makes it possible to compensate for the characteristic changes and nonlinearity of USM. The parameter-free tribes-PSO requires no information about the USM beforehand; hence its application overcomes the problem of Jacobian estimation in the conventional back propagation (BP) method of NN. The effectiveness of the proposed method is confirmed by experiments.
\end{abstract}

\section{Introduction}

The USM is a unique motor which employs frictional force generated by high-frequency mechanical vibration in a piezoelectric element as its drive source. It possesses excellent features such as compactness, low mass, noiselessness, high torque at low speed range, high retention torque, and no electromagnetic noise. Therefore, USMs are expected to be applied as actuators in industrial fields, especially, in the medical and welfare field $[1,2]$.

However, there are several drawbacks which prevent USMs from being precise positioning actuators. Firstly, it is hard to derive a plant model of USM based on physical analysis because of its friction driving principle. Although some plant models for USM have been proposed, they are too complex to apply modern control theories. Hence, the PID control which has a simple architecture and works well even without a mathematical model [3,4] has been widely used in USM control. Secondly, USMs possess characteristic variations and nonlinearity which are associated with temperature and load conditions. In order to compensate for characteristics of USM, some intelligent approaches based on NN have been proposed in recent years [5-10]. In these intelligent methods, it is not hard to find the following two facts. The conventional NN with BP method, which requires Jacobian estimated beforehand [6-10], is not easy to adopt practically. The conventional ones, such as genetic algorithm (GA) type NN [9] and PSO type NN [10] need much time on getting proper parameters of the algorithms.

Therefore, we propose the scheme of IMC-PID control combined with a tribes type NN for the position control of USM. In the proposed scheme, the tribes type NN is adopted to adjust the IMC-PID controller. Learning of NN is carried out by the tribes-PSO algorithm. Based on the classical PSO algorithm proposed by Kennedy and Eberhart [11-13], Clerc proposed a parameter-free PSO algorithm named tribes [14-16]. It is considered as an efficient configuration, which frees the user from getting proper parameters in using PSO algorithm. A modified type of the tribes-PSO algorithm for the position control of USM is introduced in this paper.

This paper is organized as the follows. The IMC-PID is introduced in Sec. 2. In Sec.3, the tribes type NN. Section 4 introduces the experimental setup. The experimental results and discussions are given in Sec. 5. Section 6 is the conclusions. 


\section{IMC-PID Control}

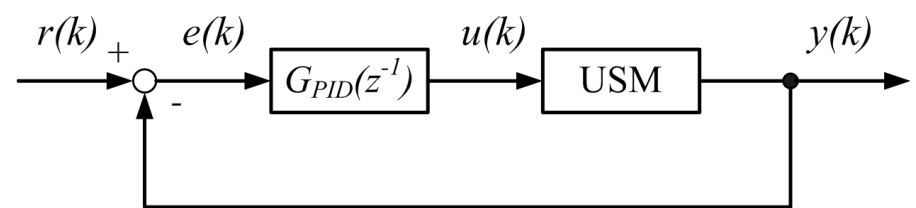

Fig. 1. Block diagram of PID control.

PID Control. As Fig. 1 shows, in the PID scheme for USM, $r(k), u(k)$, and $y(k)$ are the object input, the input and the output, respectively. $u(k)$, the output of PID, can be synthesized as

$$
u(k)=u(k-1)+\left(K_{P}+K_{I}+K_{D}\right) e(k)-\left(K_{P}+2 K_{D}\right) e(k-1)+K_{D} e(k-2)
$$

where $K P, K I$ and $K D$ are the gains of PID controller. The error denoted by $e(k)$ is expressed as

$$
e(k)=r(k)-y(k)
$$

Furthermore, the PID controller $G_{P I D}\left(z^{-1}\right)$ can be denoted as Eq. (3) shows.

$$
G_{P I D}\left(z^{-1}\right)=\frac{K_{P}\left(1-z^{-1}\right)+K_{I}+K_{D}\left(1-z^{-1}\right)^{2}}{1-z^{-1}}
$$

IMC-PID Control. The IMC-PID control requires a mathematical model of the plant. However, the mathematical model of USM is quite difficult to derive. Here we adopt a virtual plant model of USM expressed as

$$
y(k)=G\left(z^{-1}\right) u(k)
$$

where

$$
G\left(z^{-1}\right)=\frac{b_{0} z^{-1}}{1+a_{1} z^{-1}+a_{2} z^{-2}}
$$

Figure 2 shows the block diagram of the IMC scheme. $G_{I M C}\left(z^{-1}\right)$ means the IMC controller. $G\left(z^{-1}\right)$ connected to the USM in parallel is the internal model expressed as the same form in Eq.(5). $G\left(z^{-1}\right)$ can be divided into a minimum phase element $G_{M}\left(z^{-1}\right)$ and a leg time $z^{-1}$ expressed as

$$
G\left(z^{-1}\right)=G_{M}\left(z^{-1}\right) z^{-1}
$$

where

$$
G_{M}\left(z^{-1}\right)=\frac{b_{0}}{1+a_{1} z^{-1}+a_{2} z^{-2}}
$$

We define a first-order lag filter as Eq.(8) shows.

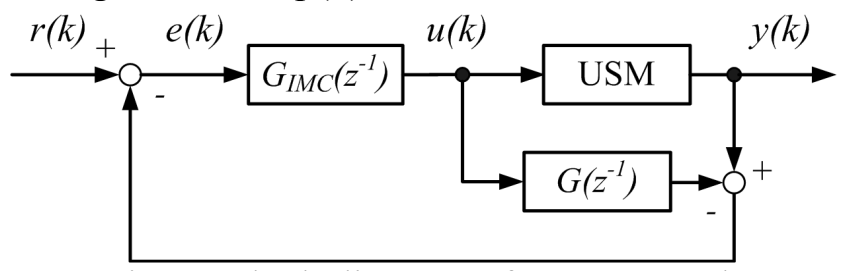

Fig. 2. Block diagram of IMC control. 


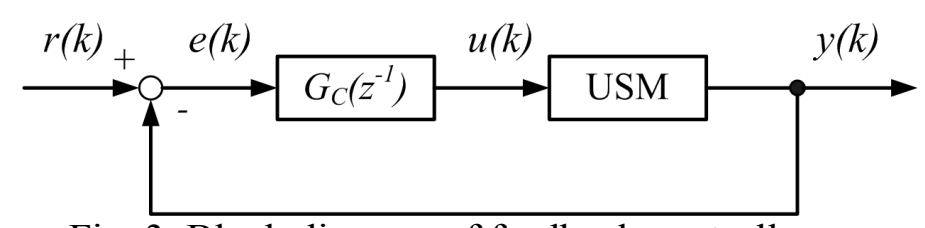

Fig. 3. Block diagram of feedback controller.

$$
f\left(z^{-1}\right)=\frac{1-\alpha}{1-\alpha z^{-1}}
$$

where $\alpha$ is a control parameter which can be decided arbitrary within the range of $0 \leq \alpha<1$. The controller $G_{I M C}\left(z^{-1}\right)$ can be expressed as

$$
G_{I M C}\left(z^{-1}\right)=G_{M}^{-1}\left(z^{-1}\right) f\left(z^{-1}\right)=\frac{1+a_{1} z^{-1}+a_{2} z^{-2}}{b_{0}} \cdot \frac{1-\alpha}{1-\alpha z^{-1}}
$$

The IMC scheme can be expressed as a feedback control structure as Fig. 3 shows. The controller $G_{C}\left(z^{-1}\right)$ can be expressed as Eq.(10) shows

$$
G_{C}\left(z^{-1}\right)=\frac{\bar{K}_{P}\left(1-z^{-1}\right)+\bar{K}_{I}+\bar{K}_{D}\left(1-z^{-1}\right)^{2}}{1-z^{-1}}
$$

where

$$
\begin{aligned}
& \bar{K}_{P}=-(1-\alpha) \frac{a_{1}+2 a_{2}}{b_{0}} \\
& \bar{K}_{I}=(1-\alpha) \frac{1+a_{1}+a_{2}}{b_{0}} \\
& \bar{K}_{D}=(1-\alpha) \frac{a_{2}}{b_{0}}
\end{aligned}
$$

Using above gains, the control input can be synthesized as

$$
u(k)=u(k-1)+\left(\bar{K}_{P}+\bar{K}_{I}+\bar{K}_{D}\right) e(k)-\left(\bar{K}_{P}+2 \bar{K}_{D}\right) e(k-1)+\bar{K}_{D} e(k-2)
$$

\section{Tribes Type NN}

Neural Network. Figure 4 shows the scheme of IMC-PID combined with tribes type NN for USM. The system parameter $\alpha(k)$ is designed to be adjusted by the tribes-NN controller. Weights of the NN are updated by the tribes-PSO algorithm to compensate for characteristics of USM.

As shown in Fig. 5, an NN controller with a two-layer structure is employed. There are four neurons in the input layer. $I_{i}(k)$ expressed in Eq.(13) is brought into $\mathrm{NN}$ as input signal.

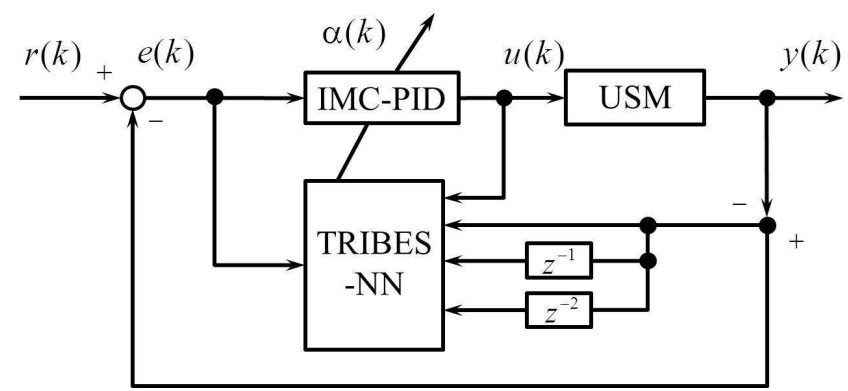

Fig. 4. Block diagram of the proposed method. 


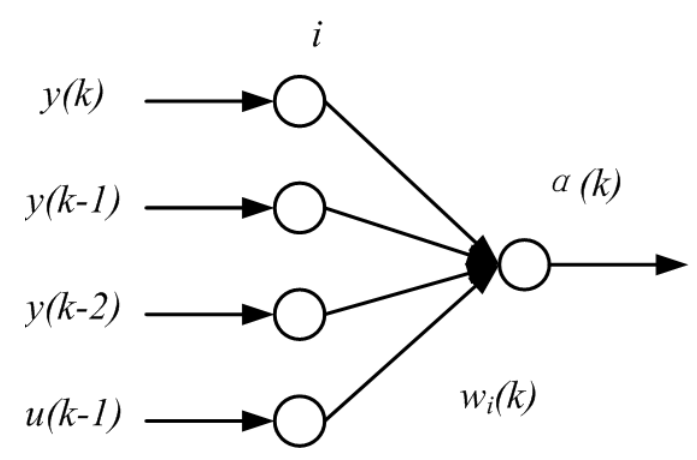

Fig. 5. Topologic structure of neural network.

$$
I_{i}(k)=\{y(k), y(k-1), y(k-2), u(k-1)\}
$$

$\alpha(k)$, the output of $\mathrm{NN}$, is used as the control parameter in IMC-PID controller. It can be estimated as the following equation shows

$$
\alpha(k)=f_{s}\left(\text { net }_{i}\right)
$$

where the function $f_{s}(k)$ means the sigmoid function as Eq.(15) shows.

$$
f_{s}(x)=\frac{1}{1+e^{-x}}
$$

$w_{i}(k)$ presents the weights, the net $_{i}$ can be calculated as

$$
n e t_{i}=\sum_{i}^{4} w_{i}(k) I_{i}(k)
$$

Tribes. PSO is quite attractive as a stochastic optimization method in recent years [11-13]. However, finding the optimal parameters for the PSO is time consuming. Therefore, a parameter free PSO named tribes has been proposed [14-16]. In this application, a tribes-PSO algorithm modified according to the characteristics of USM servo system is applied in the NN's learning. Hence there is only one of particles can be evaluated at each time. On account of the fact that the variable size of tribes is not easy to fit the servo system, we propose a PSO with two particles updated by the displacement strategies based on probability.

In the proposed scheme, the value of weights is considered as the position information contained by particles. The particles are updated by the displacement strategies shown in Table 1. The position

Table 1 Strategies of displacement.

\begin{tabular}{|c|c|c|}
\hline $\begin{array}{c}\text { Behavior of } \\
\text { the particle }\end{array}$ & Strategy & Equation \\
\hline$(+)$ & $\begin{array}{c}\text { Independent } \\
\text { Gaussians }\end{array}$ & $X_{j}=g_{j}+$ alea $_{\text {normal }}\left(g_{j}-X_{j},\left\|g_{j}-X_{j}\right\|\right), j \in[1: D]$ \\
\hline$(=)$ & Pivot & $X_{j}=c_{1} \cdot \operatorname{alea}\left(H_{p}\right)+c_{2} \cdot \operatorname{alea}\left(H_{g}\right)$ \\
$c_{1}=\frac{f i t(W)}{f i t(W)+f i t(g)}, c_{2}=\frac{f i t(g)}{f i t(W)+f i t(g)}$ \\
\hline$(-)$ & $\begin{array}{c}\text { Disturbed } \\
\text { Pivot }\end{array}$ & $X_{j}=c_{1} \cdot \operatorname{alea}\left(H_{p}\right)+c_{2} \cdot \operatorname{alea}\left(H_{g}\right)$ \\
$b=N\left(0, \frac{f i t(W)-f i t(g)}{f i t(W)+f i t(g)}\right), X_{j}=(1+b) X_{j}$ \\
\hline
\end{tabular}




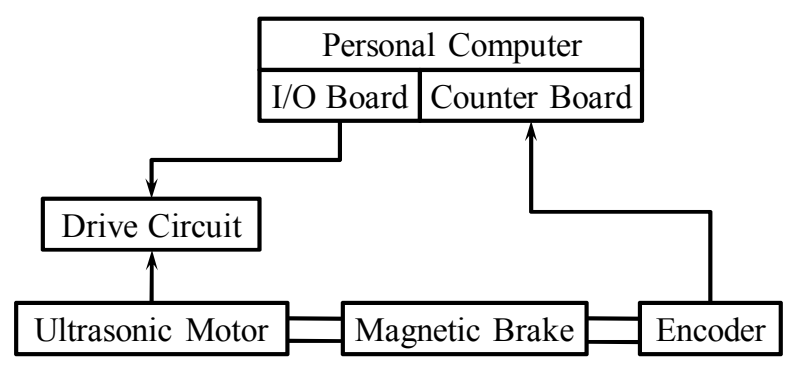

Fig. 6. USM servo system.
Table 2 Specifications of servo system.

\begin{tabular}{|c|l|}
\hline \multirow{4}{*}{ USM } & Rotation speed: $100[\mathrm{rpm}]$ \\
\cline { 2 - 2 } & $\begin{array}{l}\text { Rated torque: } 0.392 \\
{[\mathrm{~N} \cdot \mathrm{m}]}\end{array}$ \\
\cline { 2 - 2 } & $\begin{array}{l}\text { Holding torque: } 0.0392 \\
{[\mathrm{~N} \cdot \mathrm{m}]}\end{array}$ \\
\hline Encoder & Resolution: $0.0036[\mathrm{deg}]$ \\
\hline Load & $0.05[\mathrm{~N} \cdot \mathrm{m}]$ \\
\hline Sampling time & $1.0 \mathrm{~ms}$ \\
\hline
\end{tabular}

of the $n_{t h}$ particle is defined as $W_{n}=\left[w_{1}, w_{2}, w_{3}, w_{4}\right],(n=1,2)$. The best location of the $n_{t h}$ particle is $p_{n}=\left[p_{1}, p_{2}, p_{3}, p_{4}\right],(n=1,2)$. The global best location is $g=\left[g_{1}, g_{2}, g_{3}, g_{4}\right]$. fit $\left(W_{n}\right), f i t(p), f i t(g)$ mean the current fitness of the particle, particle's best fitness and global best fitness, respectively. There are three possibilities of variation for a particle: deterioration $(-)$, status quo $(=)$ and improvement $(+)$. alea $\left(H_{p}\right)$ is a point uniformly chosen in the hyper-sphere of center $p$ and radius $\|p-g\|$ alea $\left(H_{g}\right)$ is a point uniformly chosen in the hyper-sphere of center $g$ and radius $\|p-g\|$. alea $a_{\text {ormal }}\left(g-W_{n},\left\|g-W_{n}\right\|\right)$ is a point randomly chosen with a Gaussian distribution of center $g-W_{n}$ and radius $\left\|g-W_{n}\right\|[16]$.

\section{Experiments and Results}

Experimental Setup. Figure 6 shows the structure of the USM servo system. The USM, the encoder, and the magnetic brake are connected on a common axis. The position signal fetched by the encoder is input to the counter board connected to the PC. Table 2 shows the specifications of servo system.

In order to confirm the effectiveness of the proposed method, a group of experiments with the purpose of reducing the steady-state error are implemented on the system. The object input $r(k)$ is set as a rectangular signal running 30 cycles with the amplitude set from $+45 \mathrm{deg}$. to -45 degree. The period of a cycle is 4 seconds. In this paper, we call the position at +45 degree, as clockwise rotation. The opposite one at -45 degree is the counter-clockwise rotation. For the IMC-PID method, the parameters are set as $a_{1}=-0.4966, a_{2}=-0.4894, b_{0}=0.03 . \alpha$ is set as 0.7 .

In the proposed method, independent NNs are assigned to the steady-state period of clockwise and the counter-clockwise rotation, respectively. For each NN, a swarm contains two particles are employed. All the particles are initialized by random numbers from -2 to 2 . Each group of weights contained by the particles are designed to be evaluated within $50[\mathrm{~ms}]$ by the following function

$$
f i t\left(W_{n}\right)=\frac{1}{1+\cdot \sum e(k)^{2}}
$$

Results and Discussion. The responses of the last two cycles are shown in Fig. 7. There was almost no steady-state error after the learning of NN. Figure 8 shows the variation of $\alpha(k)$, the output of NN, in the first 5 cycles. The output of clockwise and counter-clockwise NNs converged to different

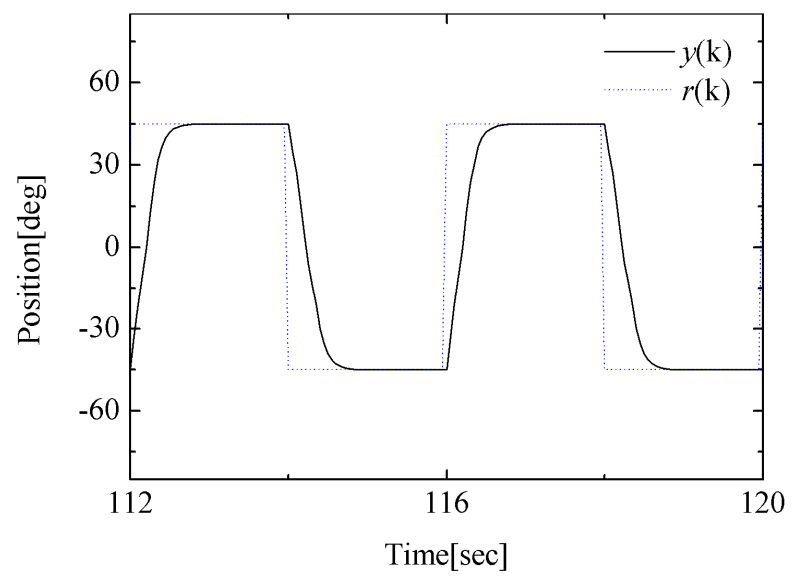

Fig. 7. Response of the proposed method.

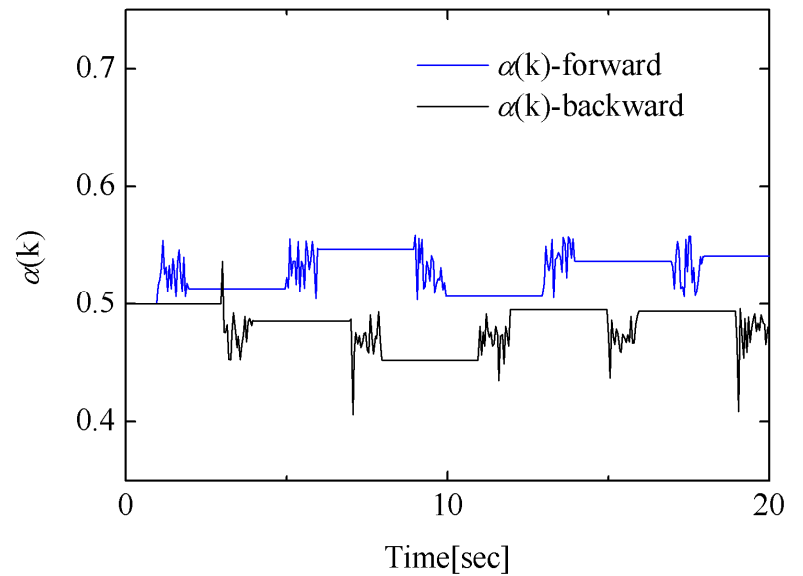

Fig. 8. The variation of the parameter $\alpha(k)$. 
value. It can be stated that the USM has the different characteristics, and the proposed method is capable to search optimal value for the characteristics of USM. Limited by the length of paper, more results will be discussed in the conference.

\section{Conclusions}

In this paper, a novel IMC-PID control combined with tribes type NN has been proposed for the position control of USM. The proposed method overcomes the Jacobian estimation problem of conventional BP type NN. The effectiveness of the proposed method is confirmed by experiments.

\section{References}

[1] T. Kenjyo and T. Sashida, An Introduction of Ultrasonic Motor, Oxford Science Publications, 1993.

[2] T. Maeno, Ultrasonic Motor, JRSJ, Vol.21, No.1, (2003) pp.10-14.

[3] J.G.Ziegler and N. B. Nichols, Optimum Settings for Automatic Controllers, Trans. ASME, Vol.64, (1942) pp.759-768.

[4] C. E. Garcia and M. Morari, Internal Model Control-1, A Unifying Review and Some New Results, Ind. Eng. Process Des. \& Dev.21, (1982) pp.318-323.

[5] T. Senjyu, H. Miyazato and K. Uezato, Position Control of Ultrasonic Motors Using Neural Network, IEEJ Transactions on Electronics, Vol.116-D, No.10, (1996) pp.1059-1066.

[6] K. Tanaka, M. Oka, A. Uchibori, Y. Iwata and H. Morioka, Precise Position Control of Ultrasonic Motor Using PID Controller Combined with NN, IEEJ Journal, Series C, Vol.122, No.8, (2002) pp.1317-1324.

[7] K. Tanaka, T. Takeguchi, J. Nakamoto, and Jinhua Li, IMC-PID Control Using NN for Ultrasonic Motor, IEEJ Trans. EIS, Vol.123, No.11, (2003) pp.1982-1988.

[8] K. Tanaka, Y. Yoshimura, Y. Wakasa, T. Akashi, M. Oka, and S. Mu, Variable Gain Type Intelligent PID control for Ultrasonic Motor, Journal of AEM, Vol.17, No.3, (2009) pp.107-113.

[9] K. Tanaka, M. Oka, Y. Wakasa, T. Akashi, and A. Naganawa, GA Adjustment Type NN-PID Control for Ultrasonic Motor, Journal of AEM, Vol.15, No.4, (2007) pp.55-61.

[10] K. Tanaka, T. Murata, Y, Nishimura, Rahman Faridah Abd, M. Oka, and A. Uchibori, Variable Gain Type PID Control using PSO for Ultrasonic Motor, Journal of AEM, Vol.18, No.3, (2010) pp. 294-299.

[11] J. Kennedy and R. Eberhart, Particle Swarm Optimization, Proc. IEEE Int. Conf. Neural Networks, Perth, Australia, (1995) pp.1942-1948.

[12] J. Kennedy, R.C. Eberhart, Swarm Intelligence, Morgan Kaufmann Publishers, 2001.

[13] M. Clerc and J. Kennedy, The Particle Swarm: Explosion, Stability, and Convergence in a Multi-Dimensional Complex Space, IEEE Trans. Evolutionary Computation, Vol.6, No.1, (2002) pp.58-73.

[14] Y. Cooren, M. Clerc, and P. Siarry, Performance Evaluation of TRIBES, an Adaptive Particle Swarm Optimization Algorithm, Swarm Intelligence, Vol. 3, No. 2, (2009) pp.149-178.

[15] Maurice Clerc, TRIBES, a Parameter Free Particle Swarm Optimizer, OEP'03, Paris, France.

[16] Yann Cooren, Amir Nakib, and Patrick Siarry, Image Thresholding Using TRIBES, a Parameter-Free Particle Swarm Optimization Algorithm, LION 2007 II, LNCS 5313, (2008) pp. 81-94. 